turn to be again replaced. All the structures and organs of the body undergo ceaseless destruction and ceaseless reparation; and the nervous system itself; the brain, the organ of thought and of volition; the cerebellum, the co-ordinator of muscular motion; the spinal cord; and the ganglia from which stimuli ase conveyed to the digestive, circulatory, and secreting organs-all are in this constant condition of transition. Particle by particle they are all being removed; particle by particle they are all being restored. When we look at man from a psychical point of view, and remember the phenomena of memory, of trains of ideas, of consecutive reasoning, of recurring sensations, how marvellous is the character which this waste and repair of nerve-tissue assumes ! Memory may be lost by a fragment of bone pressing on the cerebral convolutions, and recovered by removing the pressure. It is, there fore, exercised through cerebral matter, and is dependent for its exercise on the normal condition of that matter. But this cerebral matter is not the same at any two moments of our existence. The brain of yesterday is not the same as the brain of to-day, although the remem. brances, the hopes, the fears, the disappointments, and the successes of half a century ago, may be recalled by the sight of a once familiar object, or by the sound of the voice of a long-lost friend. How marvellous, then, must be the balance of supply and demand-the adaptation of fresh particles to take the place of effete-in this wonderful storehouse of the mind ! How exquisite must be the adaptation of the atoms which repair without a flaw the tablets of the memory !

We thus see that, in human life, the psychical and somatic functions are inextricably united. Both depend upon the pabulum assimilated by the organism for healthful activity, and fall into abeyance when the equilibrium between the forces of disintegration and renewal is disturbed. From these premisses, however, we should err if we argued their identity. The very facts which we have contemplated - the cerseless transition, activity, decay, and repair, which are taking place in the grey and white matters of the human brain-offer an insurmountable obstacle to gross materialism. We know that we are our. selves, and that we are the same as when we played at our mother's knee, although no material particle of the child we once were remain to us.

I have thus endeavoured to illustrate my subject of nerve-force in waste and supply by calling to your recollection the true functions of the nerrous system in controlling and influencing the processes of digestion and sanguification, the supply of nutrient fluid, and the process of nutrition properly so called; and, lastly, I have asked atten. tion to the processes of waste and supply in relation to the nervous system itself, and especially to the great nervous centre, the enormous development of which is that which physically differentiates man from the animals.

The subject which I have sketched out, and very feebly, I fear, illustrated, teaches much both to the clinical physician and surgeon and to the sanitarian. To the surgeon, it tells of the necessity of treating the patient, and not merely the limb-of supplying the pabulum for that nervous force which must control the processes by which wounds are agglutinated and fractures consolidated. The lessons it gives to the physician are perhaps the most important that he has to learn. One of the most distinguished of our British physicians desired that the words " he fed fevers" should be engraved on his tomb, as sufficient to confer immortality on his name, and to hand it down to the gratitude of mankind. He was right. Health is the balance between waste and supply. In disease, the balance is no longer maintained; the processes are not equal; and, although in the onset supply may be in excess, before health is restored waste will assuredly overbalance supply. To replace effete material, new elements of nutrition-new germinal matter-is necessary; and for the generation of nerve-force, which is to influence and control the reparative process, a healthy supply of renewed blood must circulate through the nervous centres. The great end and aim of practical medicine is to enable the diseased to digest and assimilate the food which alone can repair worn-out tissue and supply the nerve-force which can restore to a healthy standard disordered function.

Lastly, this subject has its lessons for the sanitarian and the politician. We hear much of education in the present day ; but what education or brain-work is possible or profitable in the case of children half starved, crowded in unhealthy dwellings, and poisoned with unwholesome water and impure air? To have the healthy active mind, we require the healthy active sensorium. Neither intellect nor virtue can be expected to flourish where squalid poverty stunts the growth, starves the brain, and nips the bud of natural affection.

To conclude : the lessons taught by a study of the relations between mind and body, from the physiologist's stand-point, have been supposed to favour a disbelief in the higher nature and destiny of man.
Never was there a more profound misconception. We have seen that physical life consists of ceaseless change. In this, the human organism is but akin to all nature, organic and inorganic. From the sun and stars, whose constitution the spectroscope has in recent years wonderfully revealed, to the grain of sand which is washed from the face of the surf-beaten rock to form again part of the bulwark of a distant shore-from the giant of the forest down to the tiny lichen hidden in the cleft of the wall-from the Leviathan of the deep down to the minutest monad--all are undergoing the same round of constant transition. Throughout the universe, as in the microcosm of man's body, the laws of disintegration and decay are balanced by the laws of repro. duction and supply. Individuals, species, genera, all pass away, and are replaced by others. Man's brain, the highest organised machine, itself follows the universal law; but man himself is not thus mutable. The ego is one and the same, from the moment it first sprang into existence at the Divine behest. That it exists unchanged by the cease. less changes of the physical organism to which it is linked, is surely evidence that it is independent of matter, and that it will exist when the present order of nature has passed and been succeeded by a phase higher and more glorious.

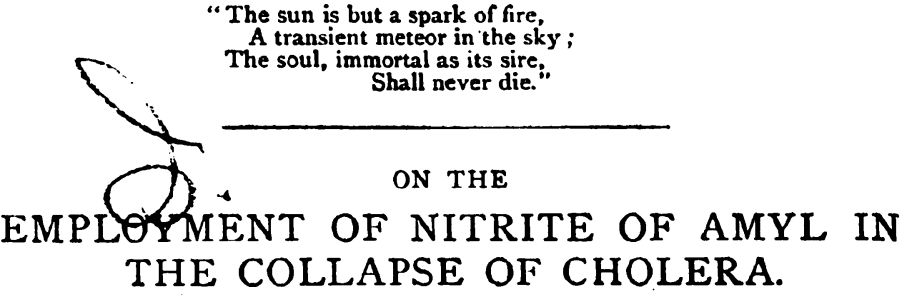

BY T. LAUDER BRUNTON, M.D., D.Sc.,

Joint Lecturer on Materia Medica, and Casualty Physician, at St. Bartholomew's Hospital ; ctc.

IN two papers which have recently appeared in this JOUR $N \mathrm{NL}$ and in the Practitioner, Dr. Tallfourd Jones very ably advocates the use of nitrite of amyl as a remedy in the collapsed stage of cholera. His proposal is, however, by no means a new one ; for it was made by Dr. Chapman in 1866 , as he mentions in a letter to this JoURNAL; and also by Dr. Gamgee about the same time. At the time that Imen. tioned the suggestion of the latter in a paper on the Action of Nitrite of Amyl in Angina Pectoris, in the Lancet for July 1867, I was una. ware that this medicine had been already tried unsuccessfully by Drs. Hayden and Cruise of Dublin. Shortly after the publication of my paper, I obtained, through the kindness of those gentlemen, a copy of their Report of the Cholera Epidemic of 1866, as treated in the Mater Misericordix Hospital, Dublin; and from this I now extract the following notices of the action of nitrite of amyl in that disease.

"When inhaled for a few minutes, he (Dr. Richardson) showed that it is capable of so exciting the circulation, that the face becomes flushed, accompanied by a thrilling sensation. It occurred to us that, in virtue of this remarkable property, the nitrite of amyl might be useful in re-establishing the circulation in the collapse of cholera. In one case, that of a man forty-two years of age, admitted in incipient collapse, the pulse rose from 102 to $\mathrm{II}_{4}$, and the temperature in the axilla rose from $95^{\frac{1}{5}}$ to $963^{\circ} \mathrm{F}$., after inhalation of the nitrite of amyl for three minutes. The inhalation appeared to aggravate his thirst. In another case, that of a woman aged 34, also in collapse, the pulse became perceptible after inhalation for a few minutes; and the patient seemed otherwise improved. Thirst seemed aggravated by the inhalation in this case also. A boy aged 4 , in collapse, inhaled the amyl for a few minutes, after which there was a slight appearance of colour in the face. The boy strenuously resisted its further administra.
tion, owing to the obstruction to free respiration which it occasioned. In several other cases the amyl was tried; but although, when inhaled for a few minutes, it usually heightened in some degree the colour of the face and surface-temperature, the difficulty of inducing patients to continue the experiment for a sufficient length of time, owing to its interference with respiration and the increased thirst which it occasioned, caused it to be abandoned."

As the results of their trial of oxygen are very interesting, I subjoin them also.

"Inhalation of oxygen by means of Dr. Richardson's apparatus was also made trial of. In one case, that of a woman in collapse, the pulse became perceptible for a few minutes. A woman aged 44, in collapse, inhaled oxygen for eighteen minutes. The pulse, previously all but imperceptible, increased in strength ; and the temperature of the surface was slightly elevated. In a third case, that of a girl aged 24 , oxygen was administered on three several occasions. The temperature rose 
slightly after the first administration; and the patient requested that she might have it again, as it gave her relief. The oxygen, like the amyl, though of temporary advantage, was found to produce no permanent benefit, and was therefore abandoned."

Both these remedies seem to offer fair promise of success, and their use is certainly indicated by the symptoms; that of nitrite of amyl by the contracted state of the vessels which is almost certainly present in the collapse of cholera ; and that of oxygen by the cyanotic appearance of the patient, and by the diminished combustion in the body, which is indicated by the small amount of carbonic acid given off from the lungs during this state. It is, therefore, rather disheartening to find from this Report that they produced no permanent benefit; and that the use of the nitrite of amyl, from which one would have been inclined to expect still more advantage than from that of oxygen, had to be abandoned, because it increased the distress of the patient by impeding still more the already difficult respiration, and rendering more intense the tormenting thirst. It is, however, better to know that they have been tried and found unsuccessful, than to cherish a false hope in their efficacy, only to be undeceived and disappointed when we come to try them ; and a careful consideration of the cause of their failure may possibly help towards a more successful plan of treatment. In order to understand why the oxygen and amyl did not fulfil the expectations which had been formed regarding them, it will be necessary to take a glance at the conditions which are found in the collapse of cholera, their probable causes, and the mode of action of the remedies em. ployed.

The most striking symptoms in the collapse of cholera are generally the excessive purging; the peculiar nature of the stools, which are liquid and colourless, resembling rice-water in appearance; and the large amount of fluid which is evacuated from the bowels. The extreme thirst of the patient, and his urgent calls for water; the shrivelled and pinched look of the surface, and its bluish colour and coldness; the cramps in the muscles; the husky voice ; and occasionally the difficult respiration, also attract immediate attention. A closer examination shows that the pulse is small, or even imperceptible; and that the proportion of carbonic acid excreted by the lungs is not more than a third of that given off in health.

In attempting to trace each of these symptoms back to its origin, we do not deal with certainties, but only with probabilities more or less strong. We cannot say with any approach to certainty what the cholera-poison is, by which all these symptoms are occasioned; but we may assign a proximate cause to each one of them - to some with a probability almost amounting to certainty; to others only with hesitancy and doubt.

The rice-water stools, which form so prominent and characteristic a symptom, have naturally attracted much attention; and numerous analyses of them have been made by Kühne, Parkes, and others, with a view to ascertain their nature and probable origin. It has thus been found that their composition is like that of the intestinal juice, except that they contain a much larger proportion of water than it does. Under ordinary circumstances, the secretion from the mucous membrane of the intestine is never so dilute as the stools of cholera ; but a very dilute juice, which is almost identical in composition with the rice-water stools, is secreted by the intestine when the nerves going to it are paralysed, as in the well-known experiment of Moreau. This gentleman took a large dog, which had not been fed for at least twenty-four hours, and, after putting it under chloroform, made an incision through the abdominal walls, and drew out a loop of intestine. Round this he put two ligatures, about four or five inches apart from one another, so that the piece of bowel between them was completely tied off from the rest of the intestine; he then cut all the nerves going to this part, but took care to leave all the blood-vessels uninjured. He next tied off in the same way a piece of intestine about the same length as the first on each side of it, but did not cut the nerves going to them. All the three parts were thus completely empty; they were all equally isolated from the rest of the intestine, and were all in exactly the same condition in regard to their vascular supply; but the nerves of the middle piece were cut, and therefore paralysed, while those of the pieces at each side of it were not. He then put the intestine back into the abdomen, and sewed up the wound. After four or five hours, he killed the dog, and examined the intestine. The two pieces which had been ligatured off from the rest, but whose nerves had not been cut, were found empty, just as they were when they were put into the abdomen; but the third piece, which lay between the two others, and whose nerves had all been divided, was found distended like a sausage with a fluid whose composition, as I have already said, was almost identical with that of cholera-stools. Now, in this experiment, all the nerves going to the intestine were cut, and therefore paralysed; but we do not know whether or not the same effects would have been produced if only some of them had been paralysed, and it is not improbable that it might have been so.

We have, then, in cholera, the same profuse secretion as in Moreau's experiment; and from identity of effect we may fairly infer identity of cause, and are, therefore, justified in concluding that the great outpouring of fluid into the bowel is due in both cases to paralysis of some, at least, of the nerves of the intestine. The probability that only some of the nerves, and not all of them, are paralysed in cholera, is strengthened by a consideration of the circumstances which induce a paralytic secretion of saliva in the submaxillary gland. Whenever a difficult question regarding secretion arises, we are always obliged to refer to this gland, because in it the process of secretion has been most fully investigated, and the conditions under which it takes place are best understood. When some of the nerves connected with the submaxillary gland are paralysed by dividing them, a continuous and profuse secretion is poured out by it. When some additional nerve-fibres, however, are divided, instead of the secretion becoming still more profuse, it becomes diminished. We do not possess sufficient knowledge regarding secretion from the intestine to be certain that it can be modified in the same way as that from the submaxillary gland; but if, as seems very probable, this analogy subsists beiween them, we can easily understand that, while a profuse flow of liquid into the intestine indicates a certain amount of paralysis of its nerves, a more moderate flow might be due to two opposite causes-viz., either to a less degree of paralysis or to a still greater one. When I speak of the choleraic discharges as a consequence of nervous paralysis, I refer only to the rice-water stools, and do not at all include the preliminary diarrhœea; for that may depend only on irritation of the intestinal nerves without paralysis, just as an abundant secretion of saliva may be obtained from the submaxillary gland by irritating some of the nerves connected with it, without division or paralysis of any one of them.

In consequence of such a large amount of water being abstracted from the blood by the intestinal glands in order to form the rice-water stools, the blood itself becomes much thicker, and the proportion of solid matter it contains much greater. The intense thirst during life, and the dryness of the tissues which is found after death, appear to bc directly due to the loss of water from the blood.

The other symptoms have also been ascribed by some persons to the effect of the thickened blood; while others attribute them partly to this, and partly to the reflex action of the intestinal lesions on the nervous system; and yet others to the direct action of the cholerapoison itself. As Dr. George Johnson has clearly pointed out, all the other symptoms can be referred to the blood being hindered from passing through the lungs. In consequence of the obstruction in front, the blood accumulates in the right side of the heart, and distends the large veins. It passes so slowly through the pulmonary vessels, that, although it is laden with carbonic acid, and is quite able to give off this gas and take up oxygen, the air which is expired contains but a small proportion of the carbonic acid which it would do in health. So little blood reaches the left ventricle, that all that it can send out at each systole hardly causes a wave in the arteries. The pulse therefore becomes weak and imperceptible, not only in the radial, but in large vessels like the brachial; and the arteries, when cut across, hardly bleed at all. The arterioles, and probably the capillaries, become empty; or, as some term it, the vital turgor disappears; and this empty state of the vessels, along with the drying of the tissues already alluded to, causes the face to become pinched and shrunken, the fingers shrivelled, and the skin to lose its wonted elasticity. From the want of any fresh blood from behind to force it on, rather than from the obstruction in front, the blood stagnates in the venous radicles, and becomes almost completely deoxygenated, giving a livid hue to the sur face ; and when a vein is opened, and the thick blood whieh distended it is emptied out, no more will flow. From the blood, which in health acts as the diffuser of heat to the extremities, and the equaliser of temperature in the body, being thus pent up in its interior, and no longer circulating through the skin or lungs, and becoming itself cooled as it warms them, the surface and the expired air both become cold, while a thermometer in the rectum may show a temperature higher than any observed even in the most intense fever.

All the symptoms are thus easily and satisfactorily explained by Dr. Johnson's hypothesis, that the circulation is obstructed in the lungs ; and even cases which at first sight might seem to militate against it, on closer examination serve, I think, only to confirm it. Such cases are those observed by Nackinnon, where, after a check had been put to the vomiting and purging, the voice, breathing, and warmth of the skin, became natural; the face had none of the peculiar character of the disease ; the patients walked about and called for food, saying that they felt well ; but their pulse was imperceptible, and in one or two days they died of coma. In these cases, it is true, the imperceptible nature 
of the pulse might be partly due to feeble astion of the heart; but it seems not improbable that it was owing in great measure to persistent obstruction in the pulmonary circulation. It would appear almost im. possible for men to live in such a condition; but Sir James Paget has shown that persons may have their pulmonary circulation obstructed to an enormous extent, and yet hardly present a symptom of anything wrong, so long as the systemic vessels contract in unison with the pulmonary ones, and do not allow any more blood to pass through them in a given time than is able to flow through the obstructed arteries in the lungs during a similar period.

While the hypothesis of obstruction to the pulmonary circulation readily explains the symptoms in collapse, it is not easy to ascertain how this obstruction is occasioned, and several causes night be assigned to it, and much might be said in favour of each of them. It might be due only to the thickened condition of the blood, which hindered it from passing through the pulmonary capillaries, although they were not contracted beyond their normal calibre, or the thickened blood itself may act as an irritant to them and cause them to contract. It is very probable that the thickened state of the blood is one cause, and by no means an unimportant one, of the retarded flow of the blood; but it appears also likely that contraction of the pulmonary capillaries is present in addition, and also that it does not depend on the condition of the blood, for, as Niemeyer and others have pointed out, the symptoms of collapse may disappear so quickly that it can hardly be supposed that time enough has elapsed to allow of the absorption of sufficient fluid to restore to the blood its normal amount of water. The blood in cholera has the colcur of bilberry-juice, and this might be supposed to indicate changes in it which would cause it to act as an irritant to the pulmonary vessels, and cause them to contract independently of any change in the amount of water it contained. This, however, can hardly be the case, as Krugenberg has found this condition continue for weeks.

Besides the causes already mentioned, there are two others to which the contraction of the pulmonary vessels might readily be attributed ; viz., reflex action from the intestines, and the direct action of the cholera poison, whatever that may be, on the vessels themselves. The occurrence of symptoms almost exactly resembling those of cholera-collapse in cases of perforation of the intestines, or of intense gastro-intestinal irritation by arsenical poisoning, would seem to favour the first view. On the other hand, the fact that there is no great lesion of the intestinal tube in cholera during life-the denudation of epithelium, on which Niemeyer laid much stress, being chiefly if not entirely a post mortem phenomena - as well as the occurrence of symptoms of collapse after intense malaria poisoning, as mentioned by Goodeve, are in favour of the second view, and between them I cannot pretend to decide. Whatever be the cause of the contraction of the pulmonary vessels in cholera, we may, I think, assume that the obstruction to the circulation is due partly to it and partly to the thickened condition of the blood.

The indications for,treatment in cholera-collapse, then, are--r. To remove the paralysis of the intestinal nerves ; 2 . To dilute the thickened blood ; 3 . To dilate the pulmonary capillaries; 4. To oxygenate the blood passing through them.

How the paralysis is to removed I for one do not know. That the copious secretion from the bowel eliminates the poison from the blood is, I think, doubtful, and any benefit that may result from it in this way is, I believe, more than counteracted by the alteration which it pro. duces in the condition of the blood. That many cases in which the discharge has been profuse have ended in recovery, and that others in which it has been scanty have terminated fatally, may simply show (if the analogy between the secretion in the intestine and submaxillary gland be correct) that the nervous paralysis has been more complete in the latter than in the former case, while the accumulation of fluid in the intestine without purging, in the so-called cholera sicca, would indicate paralysis not merely of the secreting, but also of the motor, nerves of the bowel. What $I$ have just said, however, is no argument against the use of castor-oil in the treatment of collapse. When we know so little of the pathology of the disease, or of the action of the remedies we employ, our treatment must be empirical, and successful results must be our warrant for employing one method rather than another. The second indication, viz., to dilute the blood, is generally fulfilled by giving ice and ice-cold water by the stomach. The wonderful effects which immediately follow injection of salt solutions into the veins show what an important share the loss of water from the blood has in the production of collapse. The ultimate benefit resulting from this mode of treatment has hitherto not been commensurate with the immediate relief to the symptoms which it affords; but this should only lead us to try whether we cannot, by employing different proportions of salts, or using medicines in addition to them, find some method of rendering the im. mediate effects of the injection permanent.
For the purpose of relaxing the contraction of the pulmonary capillaries, nitrite of amyl would seem, as Dr. Jones says, to be the very remedy. In this Jou RNAL for Oct. 14, Dr. George Johnson observes that nitrite of amyl may not act on the pulmonary capillaries as it does on the systemic ones. I have, however, tried it on animals, and find that it does. When given to rabbits, it causes the systemic capillaries to dilate enormously, the blood flows rapidly into the veins, and the pressure on the arterial system sinks in a corresponding degree. If it did not produce dilatation of the pulmonary as well as of the systemic capillaries, the blood which pours rapidly into the veins could not pass with equal rapidity out of them through the pulmonary vessels, and it would consequently accumulate in, and distend the right side of the heart. In order to see whether this was the case or not, I thoroughly narcotised a rabbit with chloral, put a cannula into its trachea, and kept up artificial respiration. I then opened the thorax, and, after carefully noting the appearance of the heart, passed the vapour of nitrite of amyl, mixed with air, into the lungs. When this was done, the cardiac pulsations became a little quicker, but not the slightest distension of the right side of the heart or of the jugular vein could be observed. I repeated the experiment several times with the same result. There is no reason to suppose that the chloral with which the animal was narcotised altered in any way the action of the nitrite of amyl on the pulmonary vessels, for I have found that other vapours, as well as certain poisons, injected into the jugular vein cause accumulation of blood in the ight side of the heart after the animal has been completely narcotised. I have also observed that this accumulation of blood, which I believe to be due to contraction of the pulmonary vessels caused by the poison or vapour, has disappeared in the case of one poison at least, after the injection of another drug which had been previously found to be an antidote to it in other respects. With the prosecution of these experiments I am at present engaged; and I take this opportunity of thanking Dr. Burdon Sanderson for his kindness in allowing me the use of his laboratory and apparatus.

Dr. Johnson also says that, even if nitrite of amyl were shown to possess the power of relaxing the pulmonary capillaries in health, it might not do so in disease, or at any rate not to the same extent. Some observations which I have made on its action in angina pectoris lend probability to this idea. In the paper already referred to, which I published in 1867, I mentioned my belief that angina was due to spasmodic contraction of the capillaries; and in a paper in the Clinical Society's Transactions for 1870 , I gave copies of one or two of the sphygmographic tracings which led me to form this belief. In it I noticed that occasionally after the anginal pain had disappeared from every other part of the cardiac region, it remained persistently at a spot two inches inside the right nipple. This local persistence of the pain was accompanied by a peculiar condition of the pulse, which seemed to me to indicate that the pulmonary capillaries had not become relaxed to the same extent as the systemic ones. As sphygmographic tracings, especially when taken with an instrument like the one I used in which the pressure exerted by the spring could not be estimated, sometimes admit of different interpretations, I append copies of some of them. I may mention, however, that these tracings are strictly comparable with each other, although they may not be so with tracings taken at a different time; for while these were being taken, the instrument remained attached to the arm of the patient, and the pressing of the spring which pressed upon the artery remained unaltered. In the normal condition of the patient, his pulse presented the characters seen in Fig. 3. During the attack of angina, the capillaries became contracted, and the arterial tension rose as seen in Fig $I$. When the nitrite was inhaled, the pulse in most cases quickly regained its normal character; but when the pain remained in the region of the right nipple after the inhalation, the arterial tension fell as usual, indicating dilatation of the systemic capillaries, but the volume of the pulse remained small, as shown in Fig. 2 . The small volume is, I think, due to the pulmonary capillaries not dilating, or only imperfectly, under the action of the nitrite; and if I discontinued the inhalation before the volume of the pulse became normal, as well as its character, I felt nearly certain that the spasmodic pain would speedily return with all its former intensity.

The observation of Drs. Hayden and Cruise that the pulse became perceptible in their patients after the inhalation, seems to show, however, that the nitrite did relax the pulmonary vessels during the collapse, and allowed a fuller stream of blood to pass into the left ventricle, and thence to the body. Why, then, did it increase the difficulty of breathing? One would have thought that, by allowing more blood to flow through the lungs, it would at least have alleviated this symptom, if it did not remove it altogether. The experiments of Dr. Arthur Gamgee at once enable us to answer this question. In a paper-in the Royal Society's Transactions, he showed that nitrite of amyl combines with hæmo. 
globin, the oxygen-carrier of the blood, and prevents it from giving off oxygen after it has taken it up. When the blood is exposed to the vapour of the drug as it passes through the lungs, the oxygen becomes

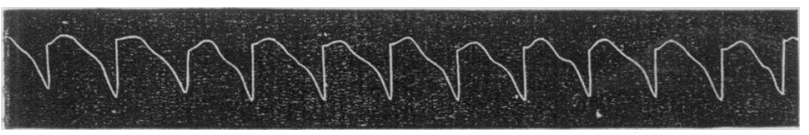

FIG. I.

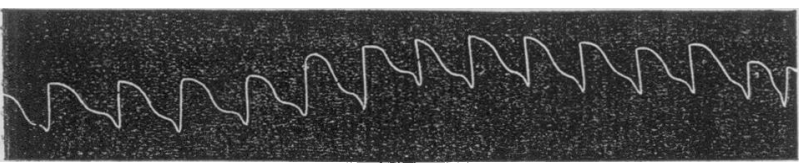

FIG. II.

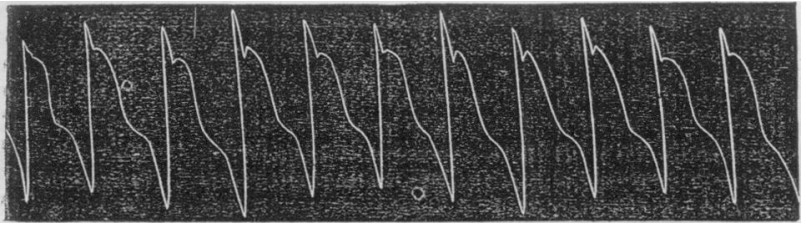

Fig. III.

locked up in it, and cannot be given off to the tissues for their use, so that however much oxygen the blood in the arteries may contain, it acts very much as if it were venous. In consequence of this, I have found that, when the vapour of the nitrite is passed into the lungs of rabbits, they are seized with suffocative convulsions. The greater part of the blood which passed through the lungs having been acted on by the vapour, it affects the brain just as it would have done if the trachea had been closed, and no air allowed to come near it during its passage through the pulmonary vessels, and therefore convulsions occur as soon as it reaches the brain. Professor H. C. Wood of Philadelphia, who has lately written a valuable paper on the action of the nitrite, found no convulsions after injecting it subcutaneously. By giving it in this way, the blood was not acted on during its passage through the lungs, and asphyxia was prevented. This, I think, shows that if nitrite of amyl is to be used at all as a remedy in cholera, it must be given in. ternally or by subcutaneous injection, and not by inhalation. This mode of administration seems to me to be indicated also in spasmodic asthma, when the arterialisation of the blood is also impaired. In angina pectoris it will act when given internally, but a larger dose is required than when inhaled; and it remains to be seen whether it can be given in cholera in sufficient doses to relax the capillaries without, at the same time, injuriously impairing the respiratory changes in the blood and tissues.

\section{MOLLUSCUM CONTAGIOSUM.}

\section{BY BALMANNO SQUIRE, M.B.}

IN reference to Dr. Liveing's note on his case of molluscum contagiosum, I may mention that some years ago a series of three cases of this disease occurring in children of one family, and affecting all three within a period of a few weeks, brought the contagiousness of the eruption so clearly before me that it occurred to me to seek a cause for it. I accordingly examined the sebaceous pellet which forms the pulp of the little tumours, with a view to discovering, if possible, the presence of a parasite; although, so far as I am aware, a parasitic nature has never been imputed to this disease.

It will be anticipated that I met at the outset with a very formidable obstacle in the well-known strong refraction of light which is characteristic of parasitic germs, and which renders it difficult to distinguish them from minute oil-globules; and that this peculiarity of fungus-spores would make it by no means easy to search for them in a sebaceous mass of which minute oil-globules are a prevailing constituent. In the diseases of the skin, the parasitic nature of which has been hitherto ascertained (favus, tinea tonsurans, and chloasma), this difficulty does not present itself. It is, therefore, easy in examining the structures (the hair and the epidermis) that are invaded by the parasites proper to these diseases, to determine the presence or absence of the fungi that are known to infest these tissues.

The difficulty which presented itself I, however, contrived to get rid of -as I think, very effectually - by digesting the sebaceous mass for many hours in ether, a process by which the mass was very considerably shrivelled. I then discovered under the microscope what I believe to have been most decided parasitic spores, to which I will not venture to give a botanical name. These spores, if my recollection serves me, were somewhat larger than those of the parasite of favus (Microsporon Audonini). I distinguished them clearly from oil-globules by a feature which I had found to be very characteristic of the large spores of the Microsporon Audonini, namely, that (unlike oil-globules) under a high power (an eighth) their apparently rounded outline, as seen under a quarter-inch power, discloses itself as a faceted (decidedly angular) outline. By this I do not mean that they have sharp angles, but rounded (as if slightly worn) angles. I did not find any mycelium; but, as regards this, I may add that I have never been able to make out any unexceptionable evidence of mycelium in hairs infested, even abundantly, with the spores of the tricophyton tonsurans, although it is plain enough in the Mierosporon Audouini, and also in the Microsporon furfur. I ought to mention, that a distinct oval nucleus, similar to what is presented under a high power by the spores of the Microsporon Audouini, was plainly perceptible also in the fungus that $I$ am imputing to the molluscum contagiosum.

There is another matter which arises from what I have put forth, and which ought not to be omitted : molluscum contagiosum is a disease which presents very anomalous and peculiar features. In no other disease of the skin do sebaceous collections increase with the surprising rapidity that is characteristic of them in this disease. In acne, in noncontagious molluscum, in sebaceous wens, the growth of the sebaceous collection itself is remarkably tardy. But the presence of a parasite will afford the requisite explanation of the difference. If, as I believe, the parasite which I have described be the special vegetable parasite of the sebaceous glands, just as the parasite present in chloasma is the parasite proper of the epidermis, and again as the parasites of favus and of tinea tonsurans are the special parasites of the hairs, - it becomes then no more difficult to understand a rapid formation of sebaceous matter provoked under the irritation of the sebaceous glands set up by the presence of a parasite, than it is to comprehend the rapid formation of scurfy epidermis produced by the irritation of the surface of the cutis by the parasite present in chloasma.

I did not publish my investigation at the time when I made it, being then busy with clinical photography; but I think the research, over which I took some pains, is worth recording, if only to serve as a hint for some more able investigator.

\section{THE USE OF BATHS IN SMALL-POX.}

By CHRISTIAN BAEUMLER, M.D., Assistant-Physician to the German Hospital.

LOokING upon hospitals as our guides in therapeutical matters, I am sure that other members of our profession besides myself must have felt some disappointment at the uncertainty of opinion regarding the above subject, which the medical evidence in the Hampstead Hospital inquiry has brought to light. The statements which were made were not characterised by that confidence which is founded on an exact knowledge whether and when a remedy is suitable or even necessary, or when it is contraindicated.

At the present day, no more doubt or uncertainty ought to exist on so important a point as this; and if it would be unreasonable to expect from any hospital accurate clinical observations on such a question at a time when an epidemic is rapidly spreading or at its height, and when necessarily all the resources of the hospital are strained to the utmost, yet the opportunity to settle this question ought not to be allowed to pass while the epidemic is declining and the hospital staffs are less overworked; for I am afraid we are still very far from the much-desired end of stamping out small-pox altogether, nor is the mortality from it so reduced as to let us lay down our hands in complacency and as to make unnecessary any further endeavours to find out means for reducing it still more.

The beneficial results which follow the use of cooling baths in other fevers can only encourage an attempt in the same direction with small-pox. Any one who has seen how the restlessness of a patient suffering from the initiatory fever of scarlatina, or in any other fever, is quieted by, and how sleep follows upon, a cool bath, cannot help thinking that the same effect would be produced in small-pox whenever the restlessness and delirium are accompanied by high pyrexia, and that, consequently, mechanical restraint would much more rarely become necessary. That the pyrexia had something to do with the delirium of those patients that had to be restrained at Hampstead, I conclude from the fact that this restraint has, in almost all the cases, only been found 